\title{
On the number of $k$-cycles in the assignment problem for random matrices
}

\author{
José G. Esteve and Fernando Falceto \\ Departamento de Física Teórica, Facultad de Ciencias and \\ Instituto de Biocomputación y Física de sistemas complejos. \\ Universidad de Zaragoza, E-50009 Zaragoza (Spain) \\ E-mail: esteve@unizar.es and falceto@unizar.es
}

\begin{abstract}
.
We continue the study of the assignment problem for a random cost matrix. We analyse the number of $k$-cycles for the solution and their dependence on the symmetry of the random matrix. We observe that for a symmetric matrix one and two-cycles are dominant in the optimal solution. In the antisymmetric case the situation is the opposite and the one and two-cycles are suppressed. We solve the model for a pure random matrix (without correlations between its entries) and give analytic arguments to explain the numerical results in the symmetric and antisymmetric case. We show that the results can be explained to great accuracy by a simple ansatz that connects the expected number of $k$-cycles to that of one and two cycles.
\end{abstract}

PACS numbers: 02.60.Pn, 02.70.Rr, 64.60.Cn

\section{Introduction}

The assignment problem (AP) for a given cost or distancet matrix $\left(d_{i j}\right),(i, j=1, \ldots, N)$ consists in finding the permutation $\sigma \in S_{N}$ that minimises the total distance $\sum_{i=1}^{N} d_{i \sigma(i)}$.

There are other problems related to this with additional constraints on the permutations allowed. Probably, the most renowned one is the traveling salesman problem (TSP) that can be formulated like the previous AP but admitting only cyclic permutations (we insist that unlike in the standard TSP our matrix does not need to be a true distance matrix). The list includes also the minimum weight simple matching problem (SMP) where only permutations composed of two-cycles are allowed (obviously in this case $N$ has to be even) and the, somehow opposite case of the minimum weight directed 2-restricted 1-factor problem (1FP), for which one-cycles and two-cycles are forbidden. If the matrix is symmetric the latter problem can be also seen as a minimum weight non directed 2 -factor problem (2FP).

From the point of view of complexity theory, it is well known (see [1]-[4]) that the TSP is NP-hard while the $2 \mathrm{FP}$ the AP and the SMP can be solved in a time the scales polynomially with $N$.

In this paper we are interested in the study of the AP for random cost or distance matrices. This problem has been studied for many years, focusing mainly on the minimal distance $D(A P)$. For example, for random matrices whose entries have probability density

\$ We use the term distance matrix although $d_{i j}$ are not necessarily true distances in a mathematical sense, in particular they do not need to be positive or symmetric. 
$\rho\left(d_{i j}\right)=\exp \left(-d_{i j}\right) \theta\left(d_{i j}\right)(\theta$ is the Heaviside step function), it was first conjectured by G. Parisi [5] and then proved rigorously ([6]-[9]) that the expected length is

$$
\langle D(A P)\rangle=\sum_{m=1}^{N} \frac{1}{m^{2}}
$$

with $N$ the number of points to be matched. Furthermore, for general random distances whose densities behave like $\rho(r)=1-a r+\mathscr{O}\left(r^{2}\right)$ near $r=0$, it is known ([10]-[14]) that

$$
\langle D(A P)\rangle=\zeta(2)-\frac{2(1-a) \zeta(3)+1}{N}+\mathscr{O}\left(N^{-2}\right),
$$

where $\zeta(x)$ is the Riemann's zeta function.

It is also known that for the TSP on symmetric random matrices with $\rho(0)=1$, the mean length of the minimal tour is ([15], [16]) $D_{0}=\lim _{N \rightarrow \infty}\langle D(T S P)\rangle=2.041 \ldots$, and the next $1 / N$ corrections are $([17],[18])$

$$
\langle D(T S P)\rangle=D_{0}\left(1-\frac{0.1437}{N}-\frac{10.377}{N^{2}}+\cdots\right) .
$$

Different probabilistic relations among the problems considered in the previous paragraphs are also well known in the literature. Namely, since the seminal work of Karp [19] we know that for purely asymmetric random matrices with uniformly distributed entries we have

$$
\lim _{N \rightarrow \infty}(\langle D(T S P)\rangle-\langle D(A P)\rangle)=0 .
$$

See also [20] and references therein for more precise estimates of this convergence.

The case of symmetric random matrices is however different, and in this situation the expected length of the solution in the TSP and in the AP do not coincide in the large $N$ limit. A different problem that has been shown to be closer to the TSP in probabilistic terms is the, above mentioned, 2FP where one-cycles and two-cycles are excluded. In ref. [21] it is shown that the expected value of the minimal distance for TSP and 2FP with symmetric random matrix coincides in the large $N$ limit. These results make clear that the structure of cycles in the optimal permutation for the AP depends strongly on the symmetry of the distance matrix and gives the clue to compare, at a probabilistic level, the different related problems.

Actually, in a recent paper [22], we found that depending on the characteristics of the distance matrix the AP can interpolate between those situations which are near the SM problem (in the sense that the optimal permutation is composed approximately of $N / 2$ cycles) and those whose optimal permutation is composed of a few cycles (just one in some cases) and one and two cycles are absent. These can be considered near the TSP or 2FP solution. The transition between both limits is governed by the correlation of the distances $d_{i j}$ and $d_{j i}$ : for positive correlations the AP problem is in the "SM regime", whereas for anti correlated distances it is "near" the TSP regime. The transition point is located where there is no correlation between the entries $d_{i j}$ (that is all the distances are independent random variables), a situation that can be solved analytically as we shall see.

In this paper we shall study the expected number of $k$-cycles in the optimal permutation and its dependence on the symmetry of the distance matrix. We shall show analytic and numerical results with special emphasis in the large $N$ limit. In particular we put into relation the probability of a permutation to be the solution of the AP with the number of one-cycles and two-cycles it contains. This ansatz can account for the numerical results with high accuracy.

The paper is organised as follows. In the next section we describe the problem with full precision. The numerical results for the expected value of the number of $k$-cycles are presented in section 3. In the next three sections we give analytic arguments to explain the 
numeric results in the three regimes: the pure random case, the antisymmetric region and the symmetric one. We finally end the paper with some comments and conclusions.

\section{Description of the problem}

Given an $N \times N$ matrix $M=\left(d_{i j}\right)$ we are interested in the permutation $\sigma \in S_{N}$ that minimises the total distance

$$
D_{\sigma}=\sum_{i=1}^{N} d_{i \sigma(i)}
$$

This problem is usually named as the assignment problem or bipartite matching problem. The novelty of our approach is that rather than looking at the minimum distance itself we focus on the permutation $\sigma$ that gives this minimum. More concretely we are interested in the number of $k$-cycles, $p_{k}, k=1, \ldots, N$ in the permutation $\sigma$ (note that this numbers, determine the conjugacy class of $\sigma$ inside $S_{N}$ ).

From this point of view we shall consider equivalent those matrices $M$ whose minimum total distance corresponds to permutations in the same conjugacy class. This implies the following equivalence relation:

$$
\begin{aligned}
& \text { i) } \quad\left(d_{i j}\right) \sim\left(\alpha d_{i j}+c\right), \quad \alpha, c \in \mathbb{R}, \quad \alpha>0 \\
& \text { ii) }\left(d_{i j}\right) \sim\left(d_{\pi(i) \pi(j)}\right) \quad \pi \in S_{N} \\
& \text { iii) } M=\left(d_{i j}\right) \sim M^{t}=\left(d_{j i}\right) .
\end{aligned}
$$

In this paper $M$ is a random matrix that depends on a constant $\lambda$, we sometimes denote it by $M_{\lambda}$, and it is constructed in the following way: take a random $N \times N$ matrix $R=\left(R_{i j}\right)$ whose entries are equally distributed, independent, real random variables with probability density $\rho$, then the entries of $M_{\lambda}=\left(d_{i j}\right)$ are given by

$$
d_{i j}=R_{i j}+\lambda R_{j i} \text {. }
$$

Note that, unlike the others, the diagonal elements depend on a single random variable and read $d_{i i}=(1+\lambda) R_{i i}$. Observe that $M_{\lambda}$ is symmetric for $\lambda=1$, antisymmetric for $\lambda=-1$ and purely random (without any correlation among its entries) for $\lambda=0$.

From the definition of $M_{\lambda}$ we have

$$
M_{1 / \lambda}=\frac{1}{\lambda} M_{\lambda}^{t}
$$

and, therefore $M_{\lambda} \sim M_{1 / \lambda}$ for $\lambda>0$ and $M_{\lambda} \sim-M_{1 / \lambda}$ for $\lambda<0$.

As it was mentioned before we are interested in the number of $k$-cycles $p_{k}$ or rather in its expected value in the distribution generated by $R$, we call it $P_{k}(\lambda)=\left\langle p_{k}\right\rangle_{\lambda}$. We shall consider $\lambda \in[-1,1]$ that ranges from the antisymmetric matrix for $\lambda=-1$ to the symmetric one for $\lambda=1$. On the other hand, given the previous equivalence $\left(M_{\lambda} \sim M_{1 / \lambda}\right.$ for $\left.\lambda>0\right)$, the results with $\lambda \in(0,1]$ repeat themselves for $1 / \lambda$. Then in an effective way we cover the whole positive real line. For the negative part things are different as we have $M_{\lambda} \sim-M_{1 / \lambda}$ for $\lambda<0$; but, if the probability density for the entries of $R$ is such that $\rho(x)=\rho(c-x)$ for some constant $c$, then the distribution of the optimal permutation with $\lambda \in[-1,0)$ is again identical to the one for $1 / \lambda$.

In the next sections we shall present the results for $P_{k}(\lambda)$ and $\left\langle n_{c}\right\rangle_{\lambda}$, where $n_{c}=\sum_{k} p_{k}$ is the total number of cycles in the optimal permutation. It is interesting to observe how they change with $\lambda$ from the antisymmetric point, $\lambda=-1$, to the symmetric one, $\lambda=1$. Different values for the dimension $N$ are considered to study the large $N$ limit. 
We also vary the distribution $\rho$ used to define the model. We mainly focus on the uniform distribution between $[0,1]$, with density $\rho_{u}$, and on the exponential one, $\rho_{e}(x)=\exp (-x) \theta(x)$. Note that $\rho_{u}(x)=\rho_{u}(1-x)$ and then, in this particular case, the interval $[-1,1]$ for $\lambda$ is enough to cover the whole real line. On the other hand, as mentioned in the previous section, $\rho_{e}$ has been extensively used in studies of the assignment problem for random matrices [5],[16] which motivates our choice.

The two distributions considered in the previous paragraph have the same limit for the density in the minimum of its support $\rho_{u}(0)=\rho_{e}(0)=1$. Many of the results obtained in the next sections hold independently of the distribution used to generate the random matrix provided its density function have a non zero limit in the minimum of its support. The same property is invoked in [5],[16] to have a minimal distance with finite limit when $N$ goes to infinity.

\section{Numerical results.}

We carried out a numerical simulation of the statistical ensemble described in the previous section. For that we generated between $10^{5}$ and $10^{6}$ random instances for $M_{\lambda}$, using the corresponding probability distributions for the elements $R_{i j}$. The number of instances depends on the dimension of the matrix, which ranges from $N=40$ to $N=1200$.

Once we generate the matrix $M_{\lambda}$ we solve the assignment problem for it using the algorithm of R. Jonker and A. Volgenant [3] and compute the number of $k$-cycles $p_{k}$ obtained in this way. In Fig. 1 1 we plot the value of $\left\langle n_{c}\right\rangle=\sum_{k} P_{k}$; there one can see the phase transition between the two regimes of $\left\langle n_{c}\right\rangle$ for $\lambda<0$ and $\lambda>0$. In the first case $(\lambda<0)$ the expected value of $n_{c}$ behaves like $\log (N)$ and is (almost) constant with $\lambda$. For $\lambda>0$ the values of $\left\langle n_{c}\right\rangle$ grow linearly with $N$ and $\lambda$ [22].

To understand the behaviour of $\left\langle n_{c}\right\rangle$ in both regimes we analyse separately the average number of $k$-cycles, $P_{k}$, as a function of $\lambda$ and $k$. In the rest of the section we present the values obtained in the numerical simulation. In the following sections we shall give a theoretical explanation of these results.

i) One cycles: In the second plot (Fig. 2) we show $P_{1}$ as a function of $\lambda$ for different values of the dimension $N$. The dots correspond to $\rho=\rho_{u}$ for dimensions 40, 200, 400, 800 and 1200. The joined plots represent the results for $\rho=\rho_{e}$ with $N=40,200$ and 1200 . We show no error bars because these are negligible.

We observe that $P_{1}$ vanishes in all cases in the left part of the diagram, it attains a common value $P_{1}=1$ for $\lambda=0$ and finally it takes a value that grows like $\sqrt{N}$ for $\lambda=1$. We finally note that the joined plots, corresponding to a different probability density $\rho=\rho_{e}$, lay very close to their respective dots (for $\rho=\rho_{u}$ ) and the fit gets better as $N$ grows.

The scaling of $P_{1}$ with $\sqrt{N}$ is shown in the inset of Fig. 2 where we plot $P_{1} / \sqrt{N}$ as a function of $\lambda$ for different values of $N$.

ii) Two cycles: In the next plot (Fig. 3) we represent $2 P_{2}$ versus $\lambda$. As in the previous case we show it for different values of the dimension and different distributions: the dots correspond to $\rho=\rho_{u}$ and the joined plots to $\rho=\rho_{e}$.

We again see that $2 P_{2}$ vanishes near $\lambda=-1$, takes the value $2 P_{2}=1$ for $\lambda=0$ and grows, in an approximately linear way, in the symmetric region, $\lambda>0$, to a value close to $N$ for $\lambda=1$. We also observe that the points corresponding to $\rho=\rho_{u}$ fit very well with those of the joined plot corresponding to $\rho=\rho_{e}$. The inset shows the linear scaling of $P_{2}$ with $N$, for $N \geq 200$.

iii) Three cycles: The situation changes drastically when we plot $3 P_{3}$ as a function of $\lambda$ in Fig. 4. The dots correspond to $N=40,200$ and 1200 for $\rho=\rho_{u}$. The joined plot represents 


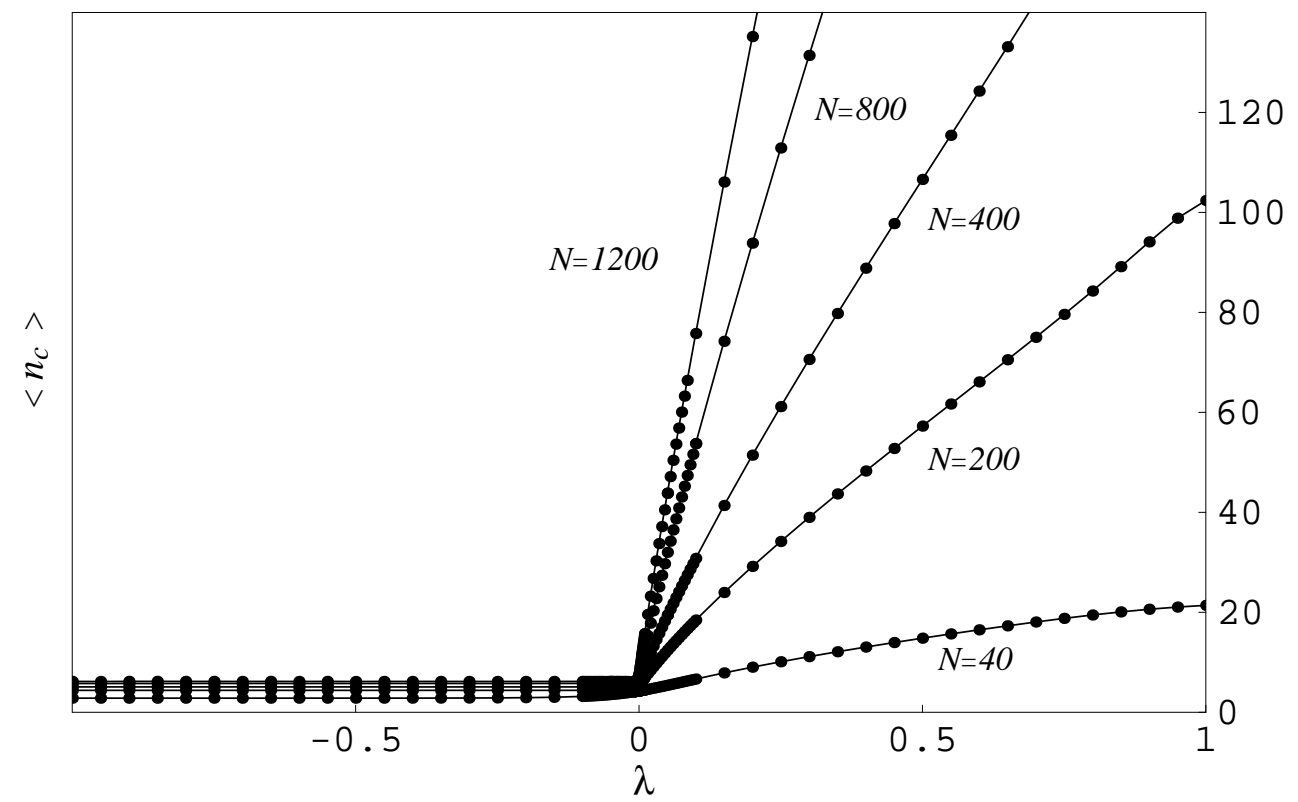

Figure 1. Mean value of the number of cycles of the optimal solution for the assignment problem at different values of $\lambda$ and $N$. The dots and the joined plots are obtained with the distributions $\rho=\rho_{u}$, and $\rho=\rho_{e}$ respectively.

the case of dimension 1200 with $\rho=\rho_{e}$.

We see that $3 P_{3}$ gets a constant value equal to 1 for almost all values of $\lambda$ and all values of $N$ and $\rho$. Only near $\lambda=1$ things depend on $N$ and as $N$ grows the value of $3 P_{3}(\lambda=1)$ tends to 1 . This limiting behaviour is common for all probability densities $\rho$.

Similar results are obtained for other odd cycles of small length compared to $N$ i.e. $5 P_{5}$ or $7 P_{7}$ are equal to 1 for all values of $\lambda$ except near $\lambda=1$, but it tends to 1 everywhere when $N$ tends to infinity.

iv) Four cycles. In the next plot (Fig. 5) we represent the behaviour of four cycles plotting $4 P_{4}$ versus $\lambda$ for different values of $N$ and $\rho$. Dots represent the values obtained for different dimensions $N=40,200$ and 1200 , all with the uniform distribution, with density $\rho_{u}$. The joined plot corresponds to $N=1200$ with $\rho=\rho_{e}$.

Comparing with the previous plot of $P_{3}$, we see no change in the left part, $\lambda<1$. However the right half is quite different. We observe that $P_{4}$ always vanishes at the symmetric point, and it follows a smooth curve (even in the large $N$ limit) from $4 P_{4}=1$ at $\lambda=0$ to $P_{4}=0$ for $\lambda=1$. A similar result is obtained for other short cycles of even length like $P_{6}, P_{8}, \ldots$ : all of them vanish at $\lambda=1$, only the shape of the curve changes, it is more horizontal near $\lambda=0$ and steeper as we approach the symmetric point.

v) Intermediate cycles: In the Fig. 6 we show the cycles of intermediate length for dimension 200 and the density $\rho_{u}$. As an example we draw $k P_{k}$ for $k=50,100$ and 150 . We see that, as in previous cases, the behaviour for $\lambda<0$ is always constant and equal to 1 . For positive $\lambda$ we see a fast transition from 1 to 0 at a value for $\lambda$ that diminishes as $k$ increases. Other intermediate values of $k$ and different values of $N$ or $\rho=\rho_{e}$ give similar results (see also Fig. 13 for odd values of $k$ ).

vi) $N-1$ cycles: In the Fig. 7 we draw $(N-1) P_{N-1}$ for $N=40,200,400$ and $\rho=\rho_{u}$. We 


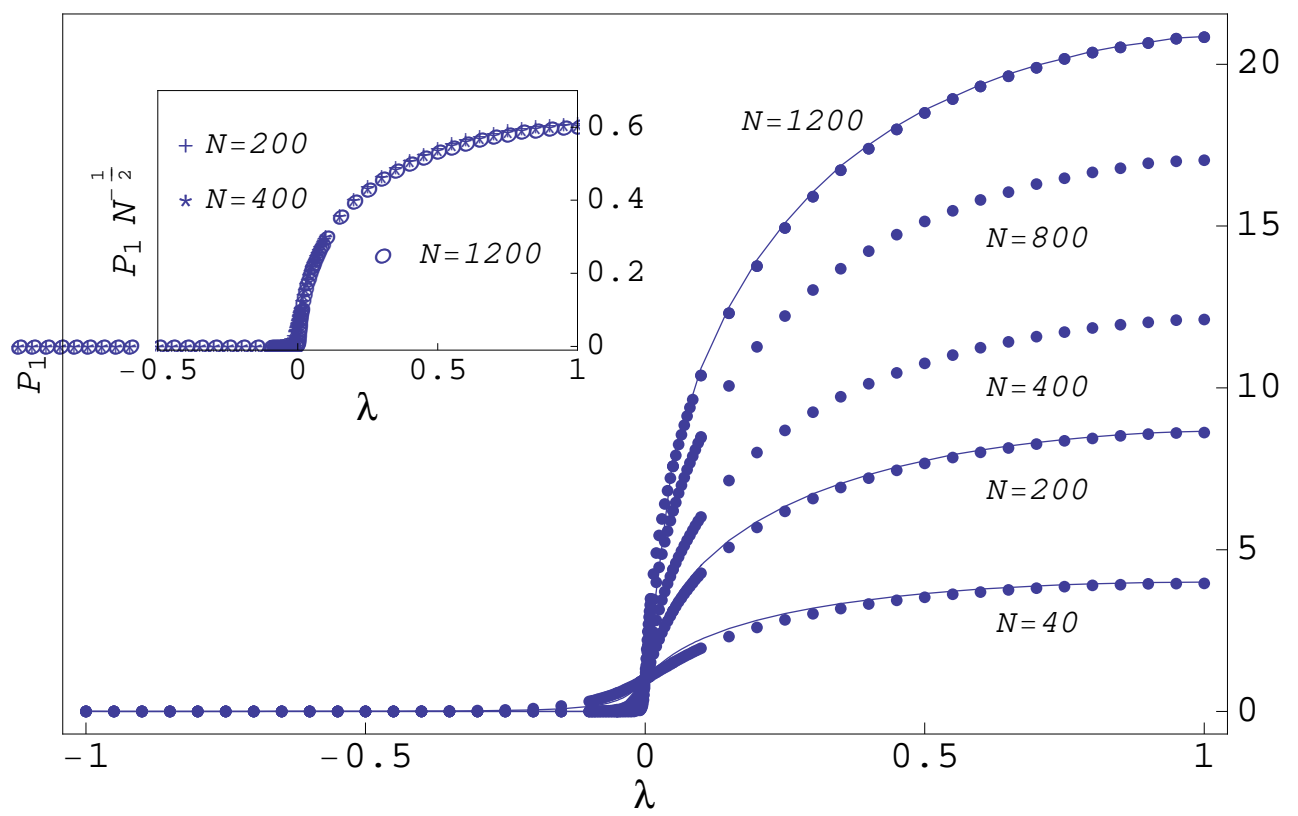

Figure 2. Average number of one-cycles in the optimal solution for the assignment problem at different values of $\lambda$ and $N$. The dots are obtained with the uniform distribution with density $\rho=\rho_{u}$, and the joined plots with $\rho=\rho_{e}$. Statistical errors corresponding to three standard deviation are not visible. The inset shows the behaviour of $P_{1} / \sqrt{N}$ as a function of $\lambda$ for different values of $N$.

see a peak, sharper as $N$ increases while its maximum moves toward $\lambda=0$. It always takes the unit value at $\lambda=0$. As before, different distributions give similar results. This plot, as well as those of $(N-2) P_{N-2}$ and $(N-3) P_{N-3}$ which are plotted in the Fig. 9 are qualitatively very different from the previous ones and also different from each other. In section 5 we shall introduce a simple ansatz that accounts for this, with great accuracy.

vii) $N$ cycles: Finally in the Fig. 8 we present the results for $N P_{N}$ for different dimensions. Note that it is again constant near $\lambda=-1$ but, contrary to the previous cases, the constant is not 1 but rather $\mathrm{e}^{3 / 2}=4.4816 \ldots$. It takes the value 1 for $\lambda=0$ and vanishes for $\lambda>0$. The width of the transition is inverse proportional to $N$. Different distributions give similar results.

To summarise the results of this section we have that for small cycles, with odd $k>2$, $k P_{k} \simeq 1$ for all $\lambda$ in the large $N$ limit. Small cycles with even $k>2$ have a smooth decay to 0 at $\lambda=1$. For cycles of intermediate length $k P_{k} \simeq 1$ from $\lambda=-1$ until it has an abrupt decay at a positive value of $\lambda$ that depends on $k$. Cycles of length close to $N$ have a very different behaviour one from each other. And finally, one and two cycles are absent for $\lambda<0$ and grow like $\sqrt{N}$ and $N$ respectively for $\lambda>1$. 


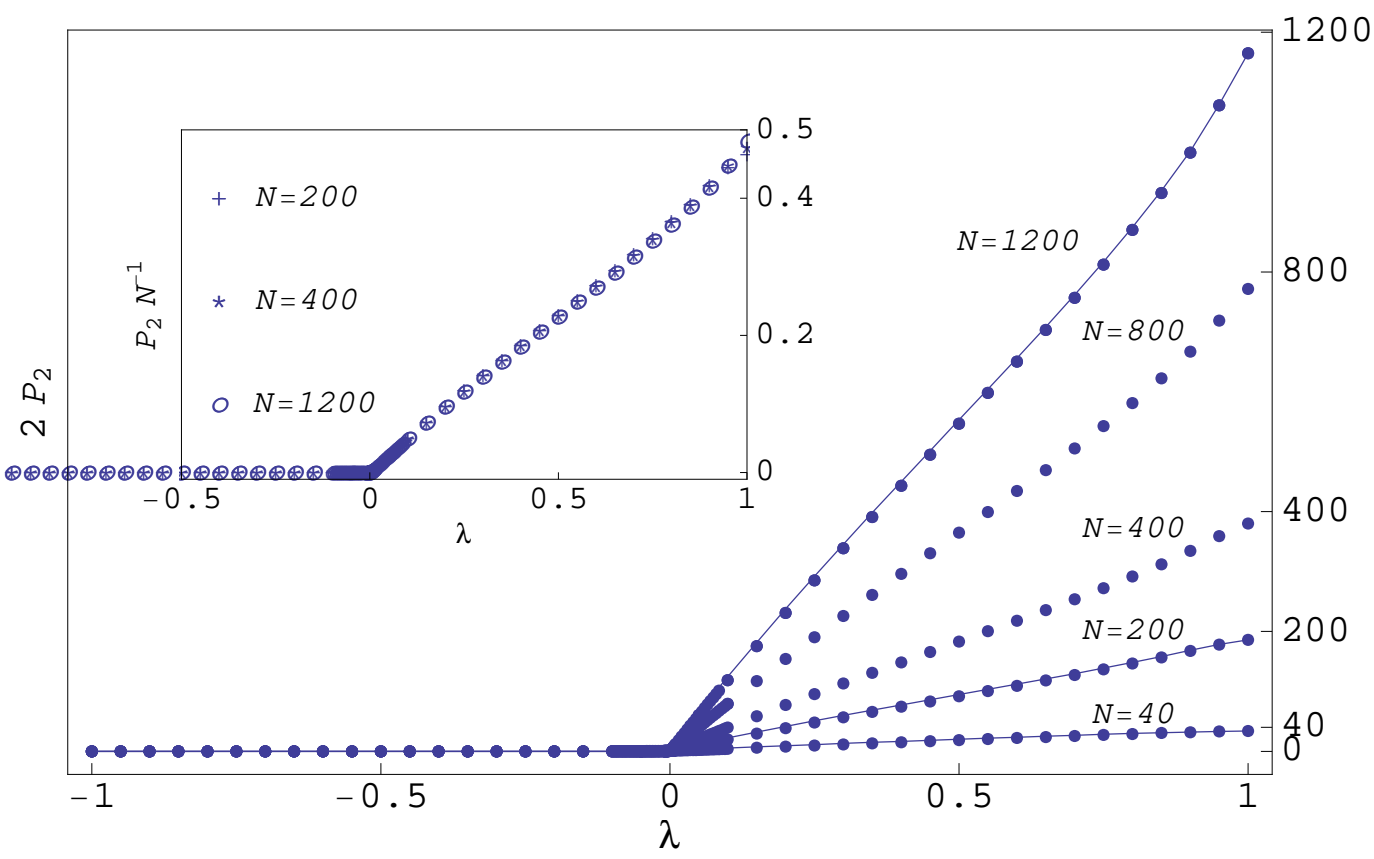

Figure 3. Average number of two-cycles (multiplied by 2) in the optimal solution for the assignment problem at different values of $\lambda$ and $N$. The results obtained with the densities $\rho_{u}\left(\rho_{e}\right)$ are displayed as points (joined plot) respectively. Statistical errors are negligible. The inset corresponds to $\frac{P_{2}}{N}$ versus $\lambda$ for different values of $N$.

\section{Solution of the model for $\lambda=0$.}

We start with the theoretical study of the model by analysing the point $\lambda=0$. In this case $M_{0}=R$ and the entries of our matrix are identical, independent random variables. Due to this fact we can show that all permutations $\sigma$ have the same probability of giving rise to the minimal distance.

The proof is very simple. Given $M_{0}=\left(d_{i j}\right)$ call

$$
\hat{\rho}\left(M_{0}\right)=\prod_{i, j=1}^{N} \rho\left(d_{i j}\right)
$$

the probability distribution in the space of matrices for $\lambda=0$. It is then clear that

$$
\hat{\rho}\left(\left(d_{i j}\right)\right)=\hat{\rho}\left(\left(d_{\pi(i) j}\right)\right),
$$

for any permutation $\pi \in S_{N}$. But if $\sigma$ is the permutation that minimises the distance $D_{\sigma}$ for $\left(\left(d_{i j}\right)\right)$ then $\sigma \circ \pi$ gives the minimum distance for $\left(d_{\pi(i) j}\right)$. It implies then that $\sigma$ and $\sigma \circ \pi$ have the same probability of being the optimal permutation, which leads to the uniform distribution in $S_{N}$

Once we have established that at $\lambda=0$ all permutations have the same probability our problem is a purely combinatorial one, and reduces to compute how many $k$-cycles there are 


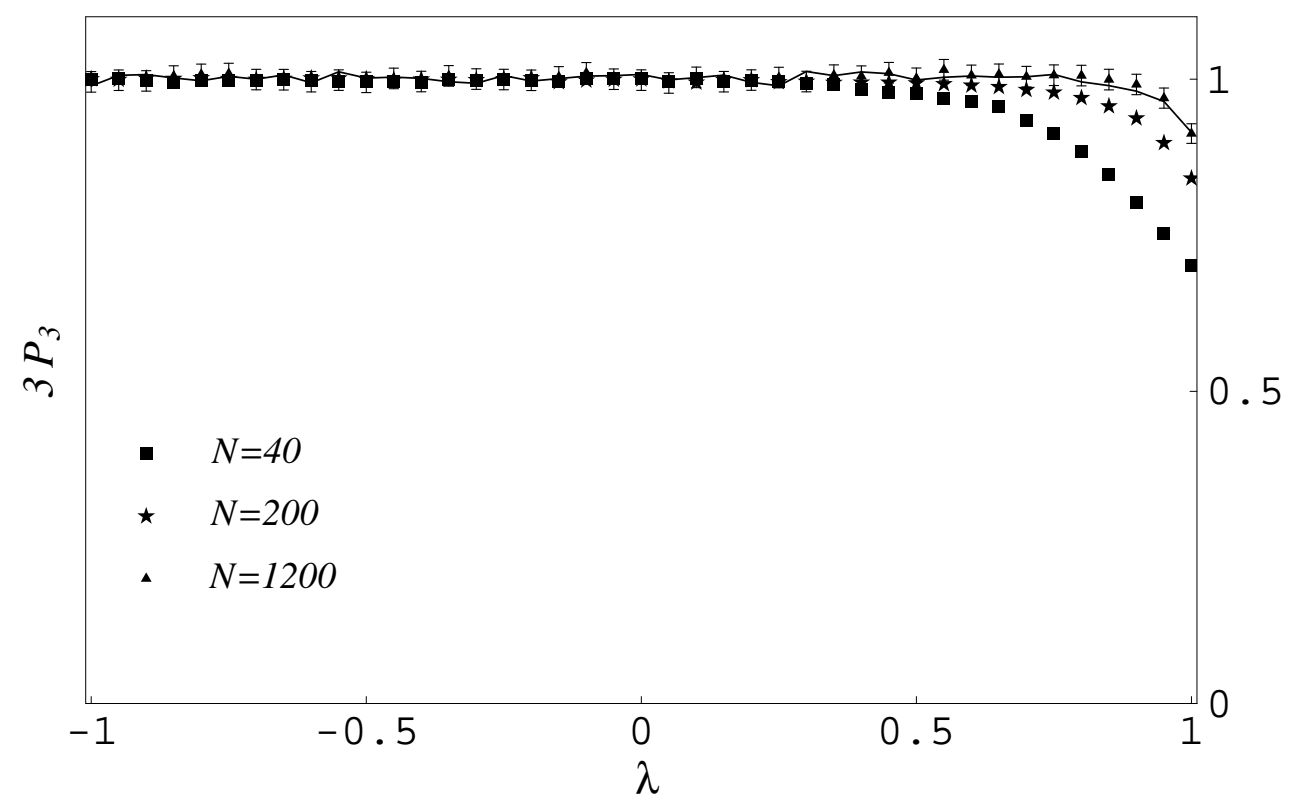

Figure 4. Average number of 3-cycles (multiplied by 3 ) in the optimal solution for the assignment problem. Symbols correspond to $\rho_{u}$ and different values of $N$ and the joint plot is for $\rho_{e}$ and $N=1200$. The error bars correspond to three standard deviations from the mean.

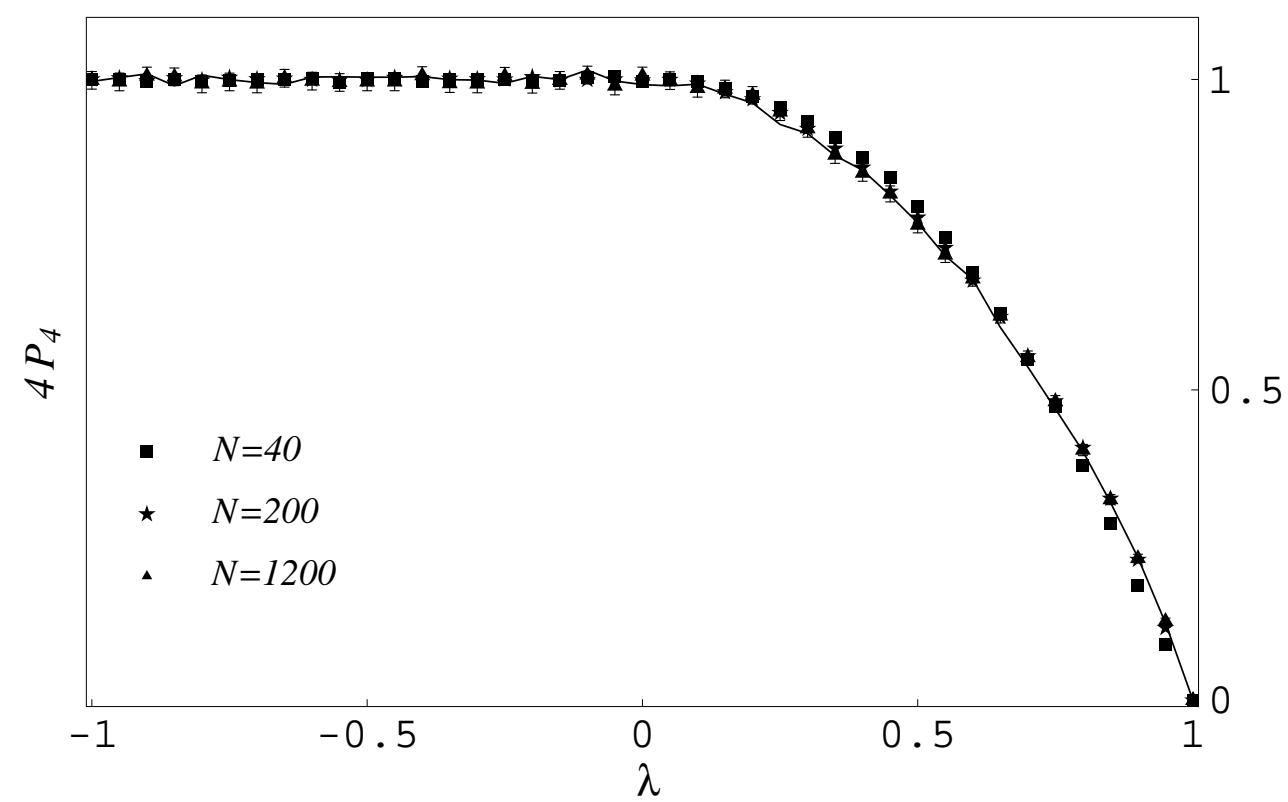

Figure 5. Mean value of 4-cycles (multiplied by 4) in the optimal solution for the assignment problem. Symbols correspond to $\rho_{u}$ and different values of $N$ and the joint plot is for $\rho_{e}$ and $N=1200$. Error bars represent three standard deviations. 


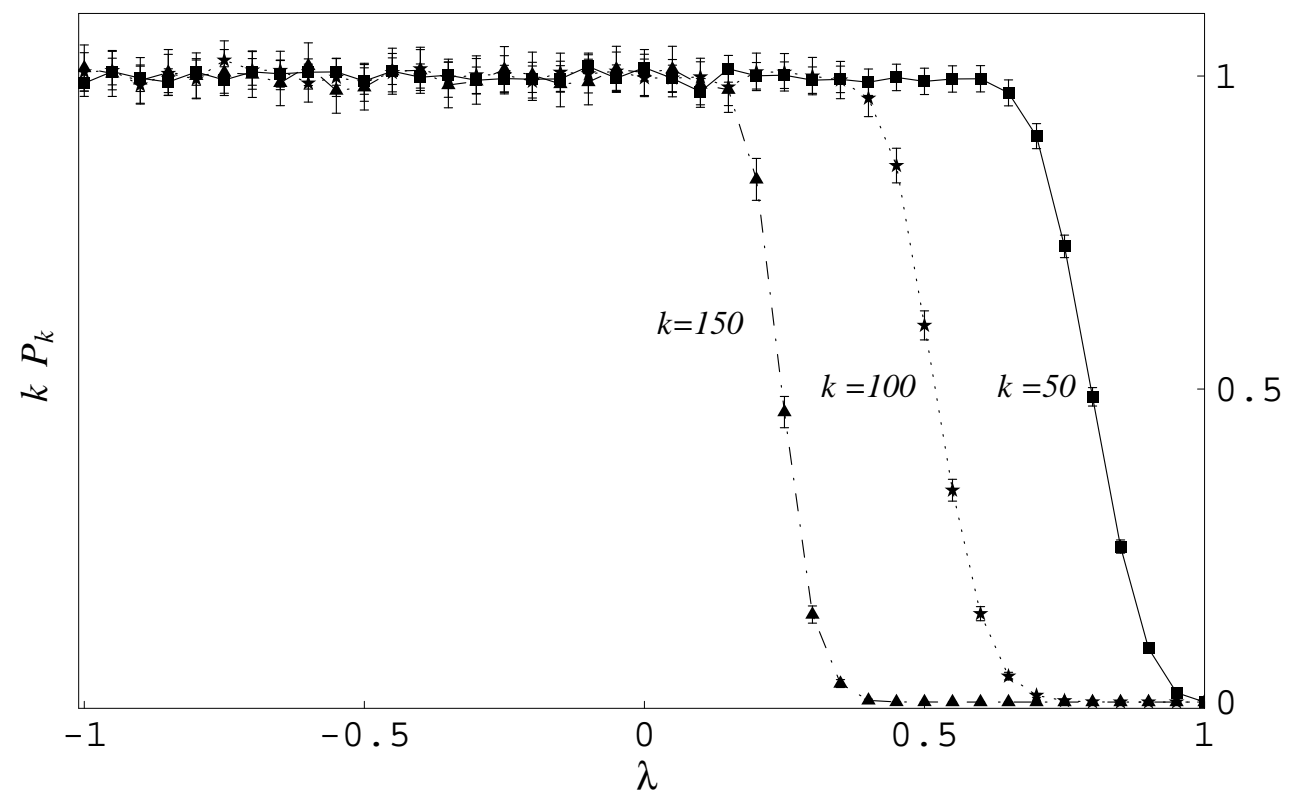

Figure 6. Average number of $k$-cycles (multiplied by $k$ ) in the optimal solution for the assignment problem at different values of $k$ and $\lambda$ for $N=200$. Error bars represent three standard deviations.

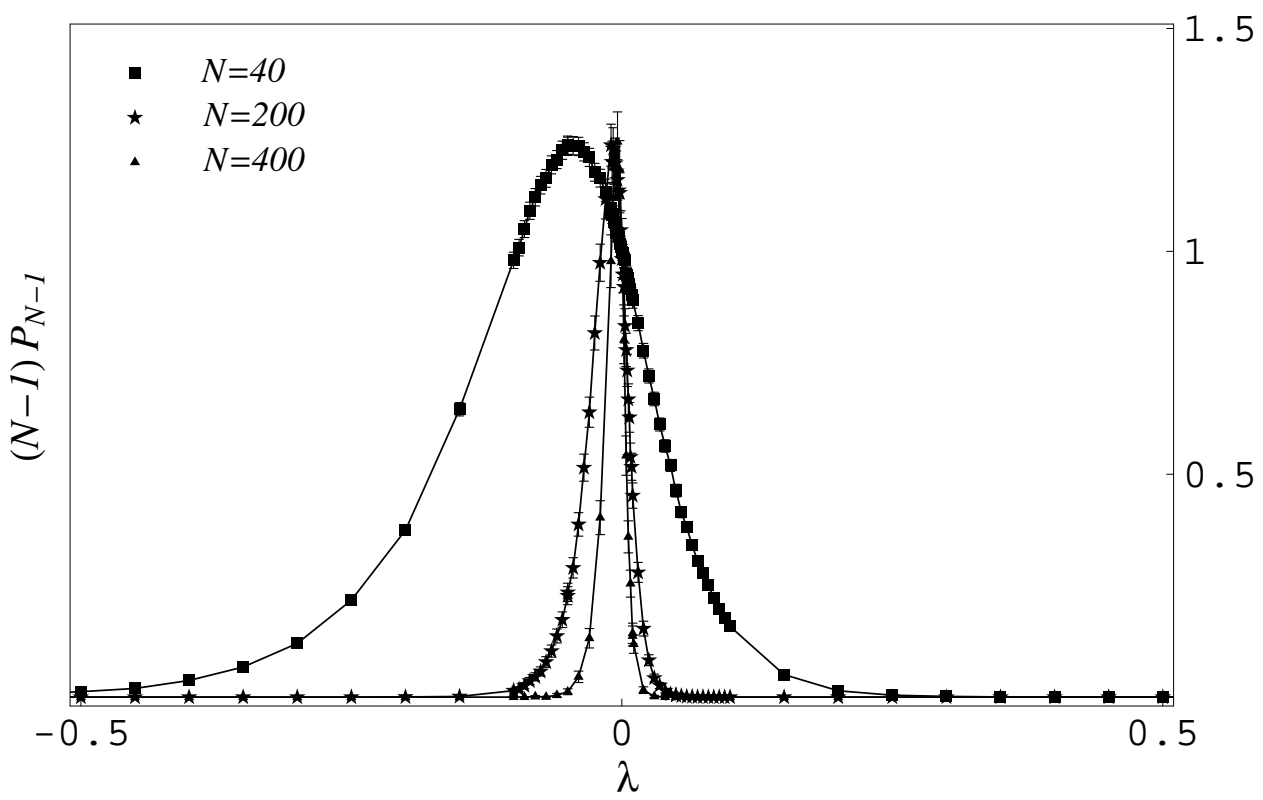

Figure 7. Average number of $(N-1)$-cycles (times $N-1)$ in the optimal solution for the assignment problem at different values of $\lambda$ and $N$. Error bars represent three standard deviations. Note the common value $(N-1) P_{N-1}=1$ at $\lambda=0$ for all values of $N$. 


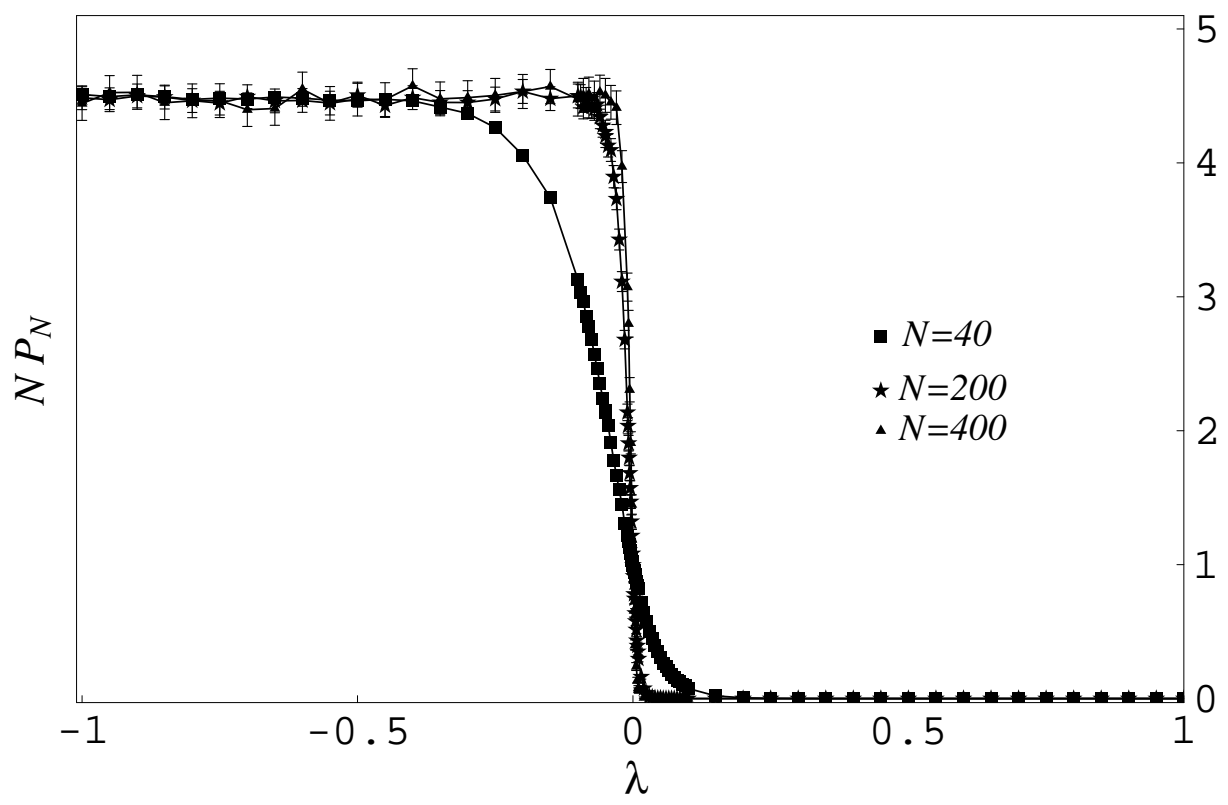

Figure 8. Average number of $N$-cycles (times $N$ ) in the optimal solution for the assignment problem at different values of $\lambda$ and $N$. Error bars represent three standard deviations.

in $S_{N}$. This number, that we call $v_{N}(k)$, is well known to be

$$
v_{N}(k)=\frac{N !}{k}
$$

as one can derive from simple counting arguments, i. e. $v_{N}(k)=\left(\begin{array}{c}N \\ k\end{array}\right)(k-1) !(N-k)$ ! where the different factors count repectively the possible choices of $k$ indexes to form the cycle, their orderings and the permutations of the rest of indexes. Note that in this way every permutation is counted as many times as the number of $k$-cycles it contains, hence the result follows. \&.

For latter purposes we shall present here a different, more cumbersome, way to derive $v_{N}(k)$ that makes use of the generating function [23],[25]. Let

$$
G(x) \equiv \sum_{m=1}^{\infty} \frac{1}{m} x^{m}=\log \left(\frac{1}{1-x}\right)
$$

be the generating function for the number of $k$-cycles in $S_{k}$ in the sense that

$$
\left.\frac{d^{k}}{d x^{k}}\right|_{x=0} G(x)=(k-1) !
$$

$\S$ We can also use the following iteration $v_{N}(k)=\left(N-k+\delta_{k 1}\right) v_{N-1}(k)+(k-1) v_{N-1}(k-1)$. The first term in the iteration counts the number of $k$-cycles that persist when one add a new index while the second term stands for the number of ways one can add a new index to a $k-1$-cycle to make it one unit larger. The $\delta_{k 1}$ is there because for one-cycles, when adding a new index linked to itself rather than to any of the preexisting ones, the number of one-cycles is increased by one 
But we rather want to compute the number of $k$-cycles in $S_{N}$. To do this we observe that the generator for the permutations in $S_{N}$ are obtained by simply taking the exponential

$$
\mathrm{e}^{G}=\frac{1}{1-x} .
$$

The procedure to obtain the number of $k$-cycles in $S_{N}$ is then simple. We introduce

$$
G_{\alpha}(x) \equiv x+\frac{1}{2} x^{2}+\cdots+\frac{1}{k-1} x^{k-1}+\frac{\alpha}{k} x^{k}+\frac{1}{k+1} x^{k+1}+\cdots
$$

so that when we take the exponential of $G_{\alpha}$ the power of $\alpha$ in every term indicates the number of $k$-cycles that the corresponding permutation contains. Therefeore, $v_{N}(k)$ is given by

$$
\begin{aligned}
v_{N}(k) & =\left.\left.\frac{d}{d \alpha}\right|_{\alpha=1} \frac{d^{N}}{d x^{N}}\right|_{x=0} \mathrm{e}^{G_{\alpha}}= \\
& =\left.\frac{d^{N}}{d x^{N}}\right|_{x=0}\left(\frac{1}{k} \frac{x^{k}}{1-x}\right)=\frac{N !}{k} .
\end{aligned}
$$

The expected number of $k$-cycles for $\lambda=0$ is then

$$
P_{k}(\lambda=0)=\frac{v_{N}(k)}{N !}=\frac{1}{k} .
$$

Note that this result is independent of $N$ and of the probability density $\rho$ we used to generate the ensemble. This explains why in all the results showed in the previous section $k P_{k}=1$ for $\lambda=0$. Finally, the expected value of $n_{c}$ is:

$$
\left\langle n_{c}\right\rangle_{\lambda=0}=\sum_{k=1}^{N} P_{k}(\lambda=0)=H_{N},
$$

where $H_{N}$ is the Harmonic series

\section{The antisymmetric region, $\lambda<0$.}

In this section we study the behaviour of $P_{k}(\lambda)$ for $\lambda<0$. We start by the observation that onecycles and two-cycles are strongly suppressed for $\lambda=-1$. The absence of one and two-cycles in the solution of the AP makes it equivalent to the corresponding $1 \mathrm{FP}$ as it was mentioned in the introduction.

This fact can be heuristically understood if one considers that the optimal permutation for $M$ comes from the choice of $N$ elementary distances $d_{i j}$ out of $N^{2}$ and, apart from the diagonal elements which are 0 , the rest of elements are half of them negative and half of them positive. Then, for large $N$, the shortest total distance will be typically obtained when we chose only negative elements and this excludes the possibility of having one-cycles $\left(d_{i i}=0\right)$ and twocycles $\left(d_{i j}=-d_{j i}\right)$ that always include non negative entries. The rest of cycles have no correlation among their elements and therefore it is reasonable to assume the equiprobability of all permutations that do not contain one-cycles or two-cycles. With this assumption we reduce the problem to a combinatorial one and we can proceed like in section 3.

Our goal, however, is to understand the expected number of $k$-cycles in the whole negative region $\lambda \in[-1,0]$ that interpolates between the absence of one and two cycles for $\lambda=-1$ to the expected values $P_{1}(0)=1$ and $P_{2}(0)=1 / 2$ at $\lambda=0$. This goal can be achieved with the following ansatz. We assume that, at least in the large $N$ limit, the 
probability for a permutation to be the shortest distance depends only on the number of onecycles and two-cycles it contains. This is consistent with the fact that only one and two cycles are sensible to the symmetry of the matrix, bonds of longer cycles are uncorrelated. Namely for a permutation with $p_{1}$ one-cycles and $p_{2}$ two-cycles the probability is proportional to $q_{1}^{p_{1}} q_{2}^{p_{2}}$, where $q_{1}$ and $q_{2}$ vanish for $\lambda=-1$ and $q_{1}=q_{2}=1$ for $\lambda=0$. The new generating function is then:

$$
G_{q_{1}, q_{2}}(x)=q_{1} x+\frac{q_{2}}{2} x^{2}+\sum_{k=3}^{\infty} \frac{1}{k} x^{k}=\log \left(\frac{1}{1-x}\right)+\left(q_{1}-1\right) x+\left(q_{2}-1\right) x^{2} / 2 .
$$

That implements the idea outlined above, as in the exponential of $G_{q_{1}, q_{2}}(x)$ every term has a weight $q_{1}^{p_{1}} q_{2}^{p_{2}}$. From this we derive the normalising factor (the total weight of the space of permutations)

$$
\Omega_{q_{1}, q_{2}}(N)=\left.\frac{d^{N}}{d x^{N}}\right|_{x=0} \mathrm{e}^{G_{q_{1}, q_{2}}}=\left.\frac{d^{N}}{d x^{N}}\right|_{x=0} \frac{\mathrm{e}^{\left(q_{1}-1\right) x+\left(q_{2}-1\right) x^{2} / 2}}{1-x},
$$

while the expected value for the number of $k$-cycles can be obtained as in previous section by introducing the factor $\alpha$ multiplying $x^{k}$ and taking the derivative of the exponential at $\alpha=1$. The result for $k>2$ is

$$
P_{k}=\left.\Omega_{q_{1}, q_{2}}(N)^{-1} \frac{1}{k} \frac{d^{N}}{d x^{N}}\right|_{x=0} \frac{x^{k} \mathrm{e}^{\left(q_{1}-1\right) x+\left(q_{2}-1\right) x^{2} / 2}}{1-x} \text { for } k>2 .
$$

To compute these quantities we use the singularity analysis approximation [24]. In the case at hand the $N^{\text {th }}$ coefficient in the power series is approximated by the residue at the pole in $z=1$. It then gives

$$
\Omega_{q_{1}, q_{2}}(N)=N !\left(\mathrm{e}^{q_{1}+q_{2} / 2-3 / 2}+\mathscr{O}\left(\left|q_{1}-1\right|^{N} / N !+\left|q_{2} / 2-1 / 2\right|^{N / 2} /(N / 2) !\right)\right),
$$

and

$$
\begin{aligned}
& \Omega_{q_{1}, q_{2}}(N) P_{k}=\frac{N !}{k}\left(\mathrm{e}^{q_{1}+q_{2} / 2-3 / 2}+\mathscr{O}\left(\left|q_{1}-1\right|^{N-k} /(N-k) !+\right.\right. \\
&\left.+\left|q_{2} / 2-1 / 2\right|^{N / 2-k / 2} /(N / 2-k / 2) !\right)(9)
\end{aligned}
$$

For small values of $q_{1}, q_{2}$ and $k$ (compared with $N$ ) this approximation can be used and we obtain $P_{k} \approx \frac{1}{k}$ for $k>2$, which is compatible with the numerical results of section 3, (see figures 4,5 and 6 ).

$P_{1}$ and $P_{2}$ do not follow the general formula but

$$
P_{1}=\left.\Omega_{q_{1}, q_{2}}(N)^{-1} \frac{d^{N}}{d x^{N}}\right|_{x=0} q_{1} x \mathrm{e}^{G_{q_{1}, q_{2}}(x)}
$$

which, in the singularity analysis approximation, gives

$$
P_{1}=q_{1}+\mathscr{O}\left(\left|q_{1}-1\right|^{N-1} /(N-1) !+\left|q_{2} / 2-1 / 2\right|^{N / 2-1 / 2} /(N / 2-1 / 2) !\right) .
$$

And

$$
P_{2}=\left.\Omega_{q_{1}, q_{2}}(N)^{-1} \frac{d^{N}}{d x^{N}}\right|_{x=0} \frac{q_{2}}{2} x^{2} \mathrm{e}^{G_{q_{1}, q_{2}}(x)}
$$

so that

$$
P_{2}=\frac{q_{2}}{2}+\mathscr{O}\left(\left|q_{1}-1\right|^{(N-2)} /(N-2) !+\left|q_{2} / 2-1 / 2\right|^{N / 2-1} /(N / 2-1) !\right) .
$$


Then for small values of $q_{1}$ and $q_{2}$ and the values of $N$ we are considering in the paper (from 40 to 1200) we can take $P_{1}=q_{1}$ and $P_{2}=q_{2} / 2$ with a very good accuracy (that covers the $\lambda<0$ region since there $q_{1}$ and $q_{2}$ are less than 1 ).

For long cycles $k \sim N$ the singularity analysis approximation is not valid any more. In this case, however, it is very easy to compute (8) explicitly. Therefore with the precision given by that of $\Omega_{q_{1}, q_{2}}(N)$ we get:

$$
\begin{aligned}
N P_{N} & \simeq \mathrm{e}^{3 / 2-q_{1}-q_{2} / 2} \\
(N-1) P_{N-1} & \simeq q_{1} \mathrm{e}^{3 / 2-q_{1}-q_{2} / 2} \\
(N-2) P_{N-2} & \simeq\left(q_{2} / 2+q_{1}^{2} / 2\right) \mathrm{e}^{3 / 2-q_{1}-q_{2} / 2} \\
(N-3) P_{N-3} & \simeq\left(1 / 3+q_{1} q_{2} / 2+q_{1}^{3} / 6\right) \mathrm{e}^{3 / 2-q_{1}-q_{2} / 2}
\end{aligned}
$$
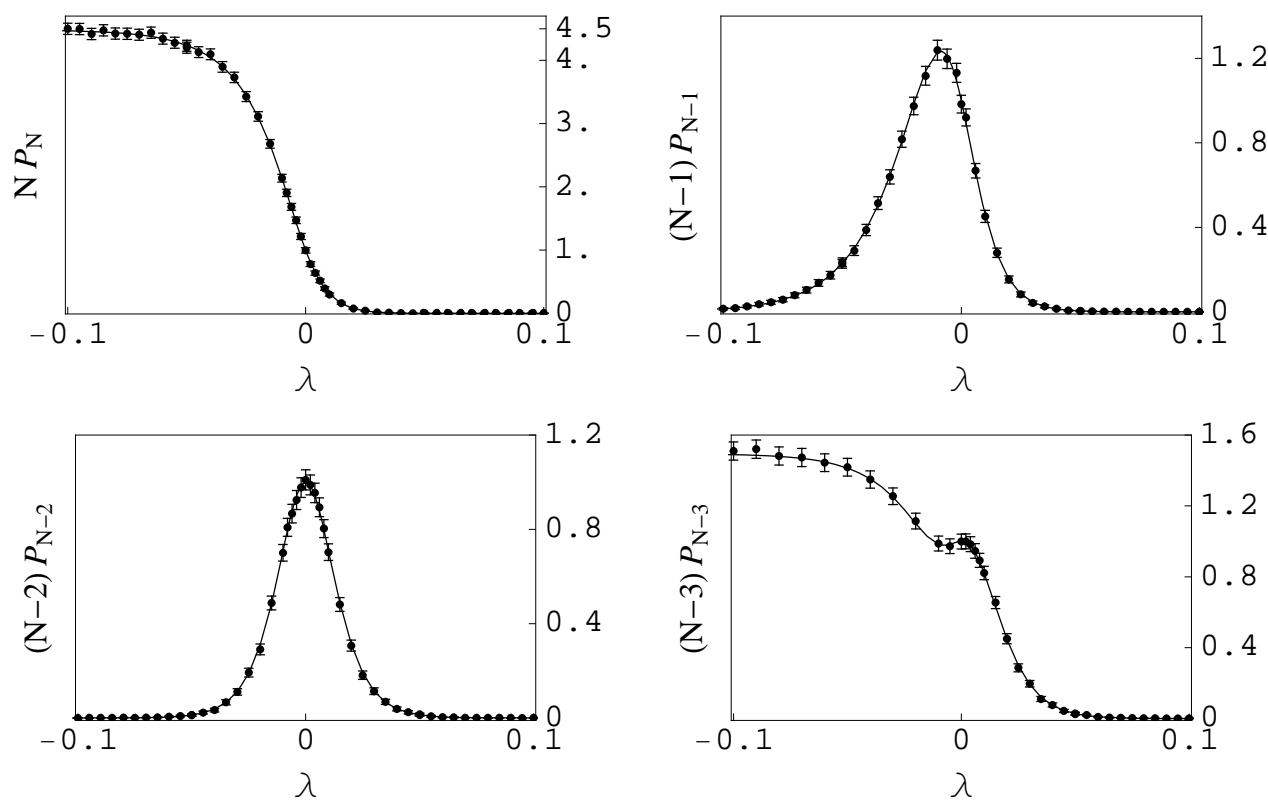

Figure 9. Average number of $k P_{k}$ (for the largest values of $k$ ) in the optimal solution for the assignment problem at different values of $\lambda$ and for $N=200$. The points are the result of our simulation and the error bars represent three standard deviations from the mean. The joined plot is the theoretical prediction using (14).

In Fig. 9 we plot $k P_{k}$ for $k=N, \cdots, N-3$ and $N=200$. The continuous line is the theoretical value obtained from (14) where we take $q_{1}=P_{1}$ and $q_{2}=2 P_{2}$. One can see that the agreement is excellent. A similar match holds for the other cases.

Thus, from the previous expressions we see that the behaviour of $P_{k}$ for for $k=1, \ldots, N$ for $\lambda \leq 0$ is completely determined by $q_{1}$ and $q_{2}$. In the rest of the section we shall study the behaviour with $\lambda$ and $N$ of this two factors. Many of the results presented below are independent on the distribution used to generate the random matrices, provided the probability density fulfils the non vanishing property in the minimum of its support that was discussed in section 2. In the rest of the paper we shall assume that this property holds.

Our first observation is the relation between $q_{1}$ and $q_{2}$ for the same value of $N, \lambda$ and $\rho$. One can check that $q_{2}=q_{1}^{2}$. A plot showing the extremely good fit between the two values as a function of $\lambda<0$ for $N=200$ and different $\rho$ is shown in Fig. 10. This relation 
can be expressed as the fact that the probability of a permutation to produce the minimal total distance, is unchanged if we change the permutation by substituting a two-cycle by two one-cycles. An argument for this comes from the fact that given two indexes $i$ and $j$, $d_{i j}+d_{j i}=(1+\lambda)\left(R_{i j}+R_{j i}\right)$ while $d_{i i}+d_{j j}=(1+\lambda)\left(R_{i i}+R_{j j}\right)$. Then both sums are identical random variables.

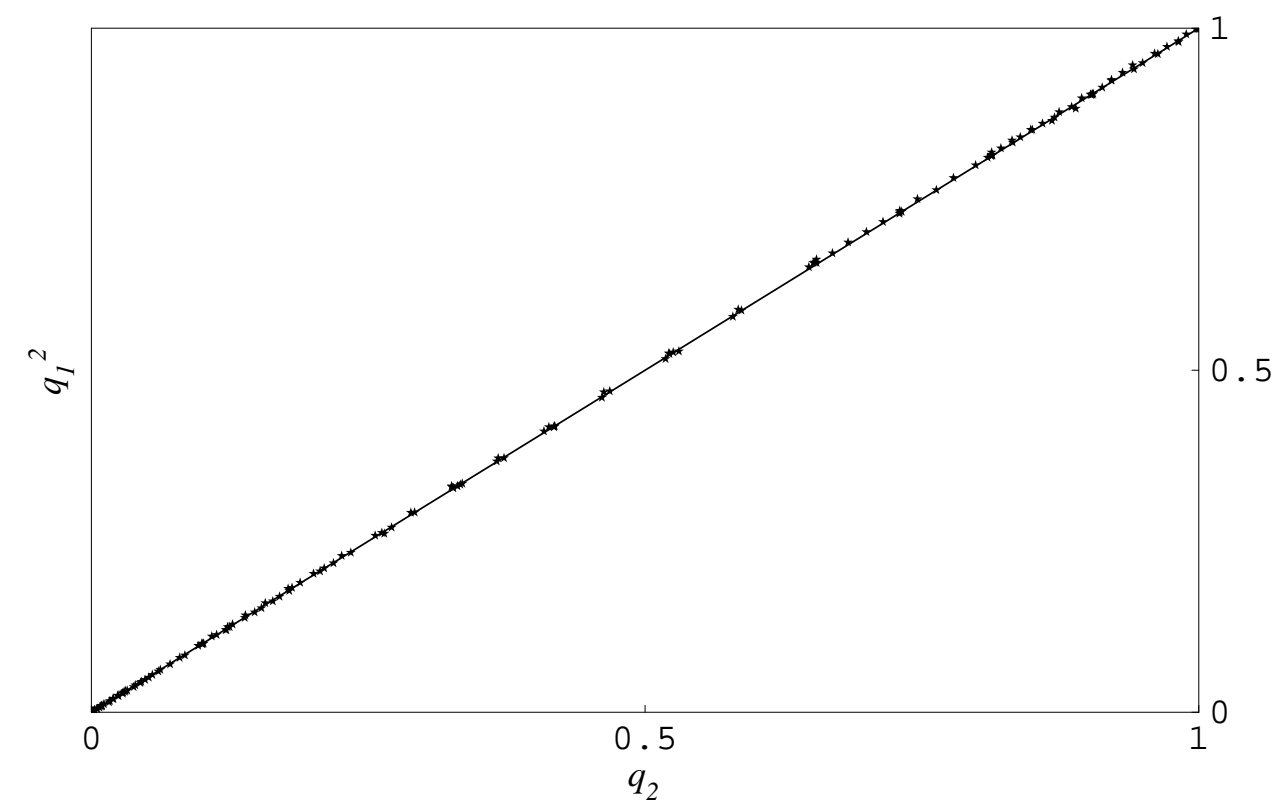

Figure 10. Values of $q_{1}^{2}$ versus $q_{2}$ for $N=40,200,800$ and $\rho=\rho_{u}, \rho_{e}$. The continuous plot is the line $q_{2}=q_{1}^{2}$.

The second important property we observe in the region $\lambda<0$ is the invariance under scaling of $\lambda$ and $N$ (see Fig. 111). In fact one can check that for a given probability density $\rho$, $q_{1}(\lambda, N)=q_{1}\left(\mu \lambda, \mu^{-1} N\right)$. And as any $P_{k}$ can be obtained from $q_{1}$ according to the formulae above, this scale invariance is true also for any $P_{k}$.

The scaling relations presented in the previous paragraph are obtained by taking a fixed probability density $\rho$ to generate the ensemble, while we change $\lambda$ and $N$. We want to examine now how $q_{1}$ depends on the distribution near the random point $\lambda=0$. Given the result that we can rescale $\lambda$ and $N$ without changing $q_{1}$ it is natural to think that $q_{1}$ can be determined by looking at only a few elements of the matrix $M_{\lambda}$. A confirmation of this conjecture is not available yet, but some partial results can be verified. Concretely we can reproduce the slope of $q_{1}$ at $\lambda=0$, that depends on the distribution, by the following formula:

$$
\frac{\partial q_{1}}{\partial \lambda}(\lambda=0, N)=\alpha N
$$

Where $\alpha$ depends solely on the distribution and is determined as follows:

for a given value of lambda fix $i \neq j$ and define $\xi_{E}=\min \left(d_{i j}, d_{j i}\right)$, also define $\xi_{D}=$ $\min \left(d_{i i}, d_{j j}\right)$. Now compute

$$
\Theta(\lambda) \equiv \frac{1}{2}\left\langle\theta\left(\xi_{D}-\xi_{E}\right)\right\rangle_{\lambda},
$$




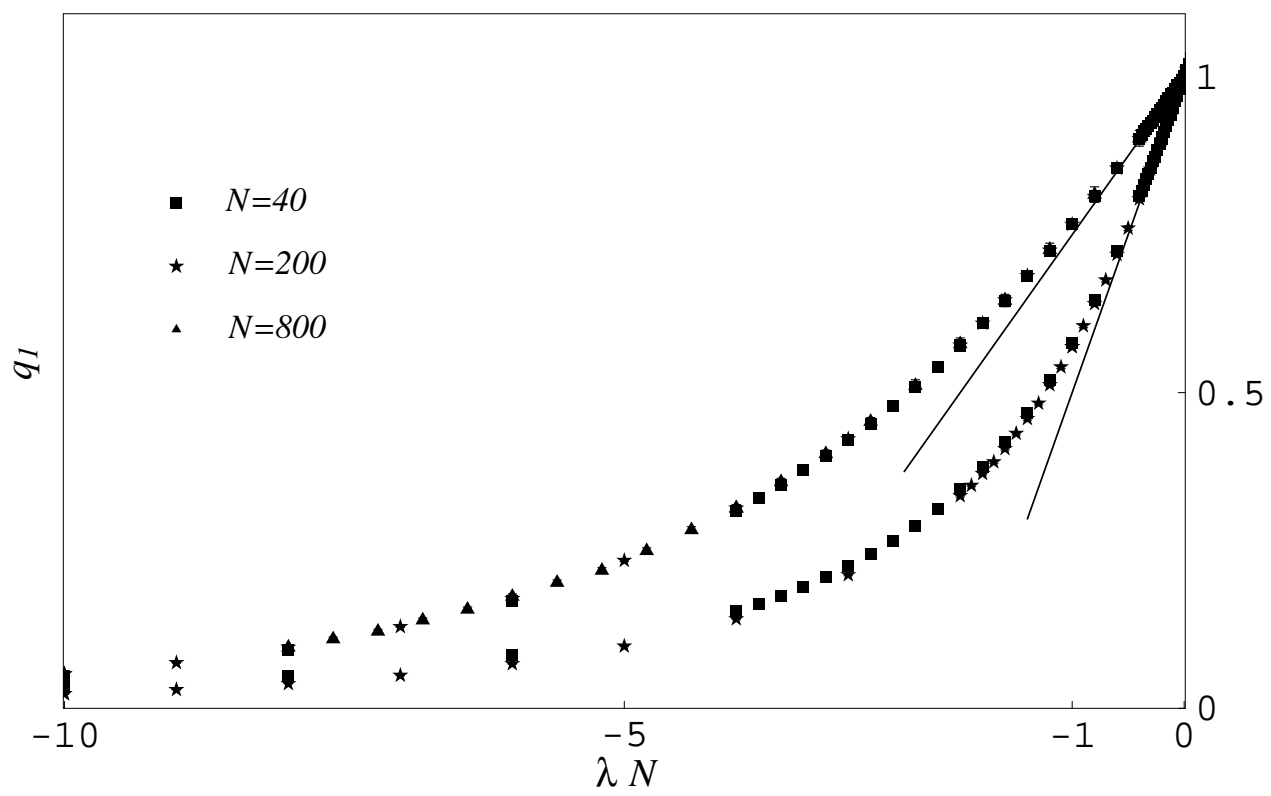

Figure 11. The points in the upper curve represent the values of $q_{1}$ as a function of $\lambda N$ for different values of $\lambda$ and $N$ and for matrices generated with probability density $\rho_{u}$. The tangent line at $\lambda=0$ is the theoretical prediction given by 15 . The lower curve is the same but for matrices generated with the exponential density $\rho_{e}$.

where with $\theta$ we denote the Heaviside step function. The coefficient $\alpha$ is obtained by

$$
\alpha=-\left.\frac{\mathrm{d}}{\mathrm{d} \lambda} \Theta\right|_{\lambda=0}
$$

As we mentioned before the value of $\alpha$ depends only on the probability density $\rho$ and can be computed with the following formula

$$
\alpha=2 \int_{-\infty}^{\infty} \rho^{2}(x) \int_{x}^{\infty} \rho(y) \int_{x}^{\infty}(z-x) \rho(z) \mathrm{d} z \mathrm{~d} y \mathrm{~d} x .
$$

The meaning of $\Theta$ is the following: it measures the probability for an extra diagonal element of a pair to be smaller than its pair and than two entries in the diagonal. It, somehow, reproduces at a small scale (only four random variables involved) the mechanism for the disappearance of one-cycles (diagonal entries) in the real problem as $\lambda$ starts to be negative. Recall that the argument for the disappearance of one and two-cycles was based in the fact that for negative $\lambda$ one of every pair of extra diagonal terms is smaller (in average) than the diagonal terms (or than half the sum of the extra diagonals). It then implies that the appearence of one and two-cycles in the optimal permutation is disfavoured. This property is quantitatively studied by means of the function $\Theta$.

Our result has been checked with different distributions and the agreement is very good. As an example we show in Fig. 11 the lines for $\rho_{e}$ and $\rho_{u}$ with slope $1 / 2$ and $1 / 4$ respectively, as obtained from (15). We can see that these lines are, as predicted, tangent to the curve of $P_{1}$ at $\lambda=0$. 


\section{The symmetric region $\lambda>0$}

As shown in Fig. 2 and 3, the first relevant fact in this region is that $P_{1}$ and $P_{2}$ grow from 1 and $1 / 2$ respectively for $\lambda=0$, to values proportional to $\sqrt{N}$ in the first case and to $N$ in the second for $\lambda=1$. A first attempt to account for this behaviour is to adjust the corresponding parameters $q_{1}$ and $q_{2}$ to fulfil equations (10) and (12), (note that now $q_{1}$ and $q_{2}$ can be $\gg 1$ so the terms of order $\left(q_{1}-1\right)^{(N-2)} /(N-2)$ ! and $\left(q_{2} / 2-1 / 2\right)^{N / 2-1} /(N / 2-1)$ ! can be important). The values of $q_{1}$ and $q_{2}$ obtained in this way are used to compute $P_{k}$ for different values of $\lambda$.

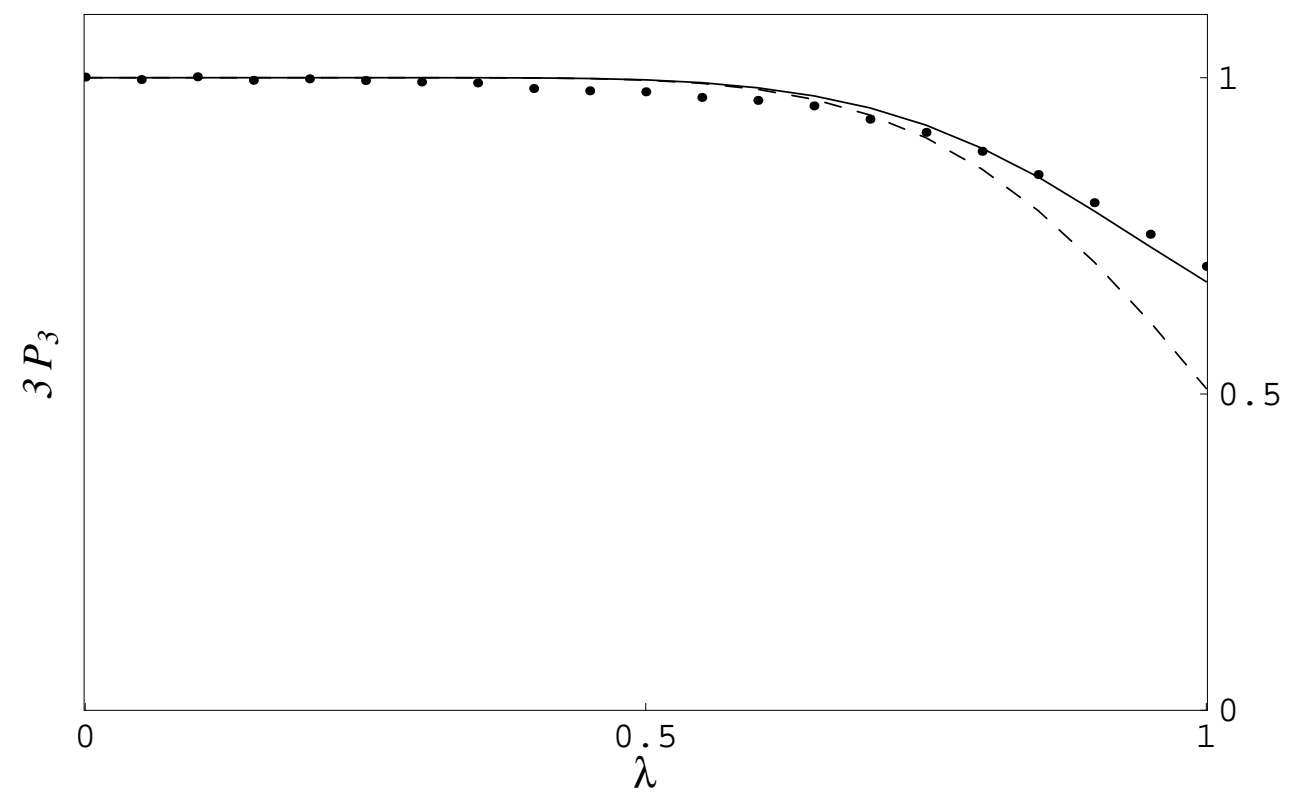

Figure 12. Numerical value of $3 P_{3}$ (dots) and the theoretical prediction using equations (10) and (12) with the corrected values of $q_{1}$ and $q_{2}$ (continuous line) and without the corrections (discontinuous line).

This procedure, however, fails to predict the numerical results in two different aspects. First, if we try to fit $P_{3}$ we obtain a large deviation with respect to the numerical value near the symmetric point. This is shown in Fig. 12 where the dots represent the numerical value and the dashed line represents the theoretical prediction obtained as outlined above. Also the disappearance of even cycles at $\lambda=1$, as shown in fig. 5, is not taken into account within this approximation i. e. the theoretical value for $P_{4}$ does not vanish at $\lambda=1$. These two facts happen to be connected and will be discussed in the next paragraph.

Is is first important to understand why even cycles disappear when $\lambda=1$. The reason is very simple, for if we had a cycle of even length i. e. $\sigma\left(i_{m}\right)=i_{m+1}, m=1, \ldots, 2 L+1$, with $i_{m} \neq i_{m^{\prime}}$ except $i_{1}=i_{2 L+1}$, then either the links in odd position $d_{i_{2 l-1} i_{2 l}}$ or those in even position $d_{i_{2 l} i_{2 l+1}}$ have a smaller sum. Assume that

$$
\sum_{l=1}^{L} d_{i_{2 l} i_{2 l+1}}<\sum_{l=1}^{L} d_{i_{2 l-1} i_{2 l}}
$$

then the new permutation $\sigma^{\prime}$ which is equal to $\sigma$ except for $\sigma^{\prime}\left(i_{2 l+1}\right)=i_{2 l}, \quad l=1, \ldots, L$ gives 
a smaller total distance. To see this, it is enough to realise that, given that $M_{1}$ is a symmetric matrix, the sum of the odd links for $\sigma$ is replaced by that of the even links in $\sigma^{\prime}$ which lowers the total distance. Hence it is impossible to have cycles of even length larger than two, in the optimal permutation of a symmetric distance matrix.

The mechanism for disappearance of even cycles we outlined in previous paragraph can be stated by saying that $2 L$-cycles break into $L$ two-cycles. This is the key point behind the improvement of the approximation in order to account for small cycles. The idea is that in equations (10) and (12) instead of using the value of $P_{2}$ obtained in the numerical simulations we subtract to it the two-cycles that come from what would be cycles of even length. The procedure is then clear: we start with a value for $q_{1}$ and $q_{2}$, say $P_{1}$ and $2 P_{2}$, we compute with this values the theoretical number of cycles of even length and subtract from it the real one obtained in the numerical simulations. These are the cycles that break into a number of two cycles. We subtract this number from $P_{2}$, introduce the new value of $P_{2}$ into equation (12) and compute again $q_{1}$ and $q_{2}$. The procedure is iterated until the desired convergence is reached. In practise in 4 or 5 iterations we obtain a very good precision.

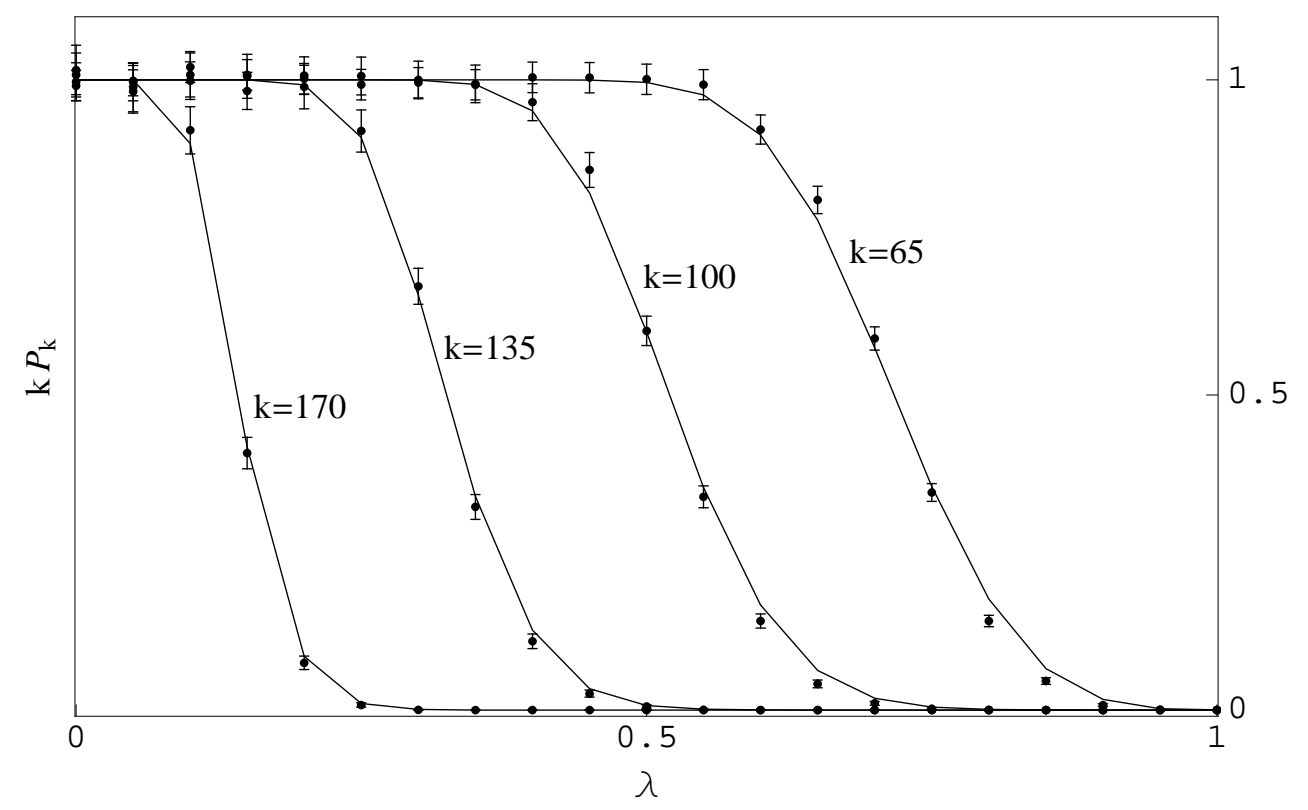

Figure 13. Values of $k P_{k}$ for intermediate values of $k, N=200$ and $\lambda>0$. The continuous line is the theoretical prediction.

In Fig. 12 we plot the numerical values for $3 P_{3}$ (dots) and the theoretical curves using the uncorrected version for $q_{1}, q_{2}$ (dashed line) and the corrected ones (solid line). We see that the fit is much better in the second instance. The theoretical prediction can be also applied to the intermediate cycles as shown in Fig. 13. The theoretical and numerical values for $k P_{k}$ with $N=200$ using the corrected $q_{1}$ and $q_{2}$, show a very good agreement.

Our last point is the relation between $q_{1}$ and $q_{2}$ that extends for positive values of $\lambda$ the fit shown in fig. 10 We find that the dependence changes in this case. A very good fit is obtained by taking

$$
q_{2}=\mathrm{e}^{\lambda} q_{1}\left(q_{1}-\lambda\right) \equiv F\left(q_{1}\right) .
$$

As it is shown in fig. 14 the agreement is rather good and it gets better in the large $N$ limit. 


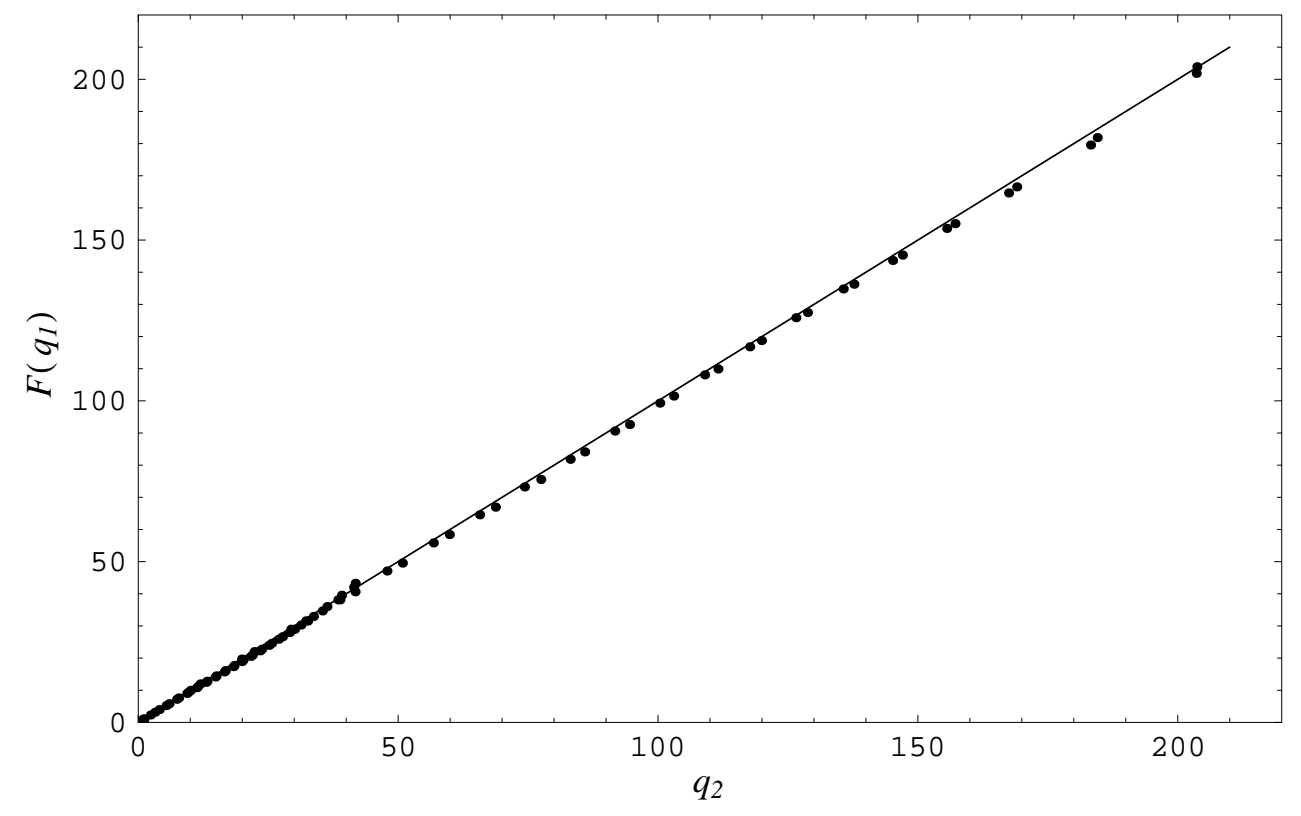

Figure 14. Values of $q_{2}$ versus $F\left(q_{1}\right)=\mathrm{e}^{\lambda} q_{1}\left(q_{1}-\lambda\right)$ for positive $\lambda$. The plot includes the points obtained for $N=40,200$ and with the probability density $\rho=\rho_{u}$ and $\rho=\rho_{e}$.

\section{Conclusions and outlook.}

The expected number of $k$-cycles in the optimal permutation of the assignment problem for random matrices, can be understood to great accuracy in terms of only two parameters, $q_{1}$ and $q_{2}$ associated to one and two-cycles. More precisely, the ansatz is that in the large $N$ limit the probability for a permutation to be the solution of the $A P$ is proportional to $q_{1}^{p_{1}} q_{2}^{p_{2}}$, with $p_{1}, p_{2}$ the number of one and two-cycles of the permutation respectively. The ansatz can be substantiated by considering that with the cost or distance matrices used in the paper only one and two-cycles are sensible to the symmetry of the matrix, as bonds of longer cycles are uncorrelated. On the other hand in the large $N$ limit we can consider the occurrence of short cycles as independent events.

With this ansatz we are able to explain, with great accuracy, the expected number of $k$-cycles in the solution of the AP for cost matrices ranging from the symmetric to the antisymmetric one. The parameters suffer an abrupt transition (in the large $N$ limit) when moving from a matrix mostly symmetric $(\lambda>0)$ to another one mostly antisymmetric $(\lambda<0)$.

We also find some universal scaling relations in the variables which are valid in the antisymmetric region. Based in this scaling behaviour we are able to give a theoretical prediction for the slope of $q_{1}$ at the critical point, $\lambda=0$.

An open problem is to understand the behaviour of the cycles of even length in the symmetric region. It is clear that, as it is argued in the paper, all of them (except the two cycles) should be absent at the symmetric point $(\lambda=1)$, but for the moment we do not know how to explain the curves that the average number of even cycles follow to reach the zero value. Finally, it would be nice to have a full theoretical study of the model (or a reliable approximation to it) that could explain the facts mentioned above. 
Acknowledgements: Research partially supported by grants FIS2006-01225 and FPA200602315, MEC (Spain).

\section{References}

[1] C. H. Papadimitriou and M. Yannakakis, The traveling salesman problem with distances one and two. Mathematics of Operations Research, 18:1-11,1993.

[2] D. Hartvigsen, Extensions of Matching Theory, Phd. thesis, Department of Mathematics, Carnegie Mellon University, Pittsburgh, PA, 1984

[3] R. Jonker and A. Volgenant, A Shortest Augmenting Path Algorithm for Dense and Sparse Linear Assignment Problems, Computing 38, 325 (1987).

[4] L. Lovász and M. D. Plummer, Matching Theory, North-Holland Publishing Co., Amsterdan, 1986

[5] G. Parisi, A conjecture on random bipartite matching, cond-mat/9801176 (1998).

[6] V. J. Dotsenko, Exact solution of the random bipartite matching model. J. Phys. A 33, 2015 (2000).

[7] D. J. Aldous, The $\zeta(2)$ Limit in the Random Assignment Problem. Random structures Algorithms 18, 381 (2001).

[8] S. Linusson, and J. Wästlund, A Proof of Parisi's Conjecture on the Random Assignment Problem. http://arXiv.org/abs/math/0303214 (2003).

[9] C. Nair, B. Prabhakar and Sharma M., A Proof of the Conjecture due to Parisi for the finite Random Assignment Problem, available at http://www.stanford.edu/ balaji/rap.html

[10] M. Mézard and G. Parisi, Replicas and optimization. J. Physique Lett. 46, L-771 (1985).

[11] M. Mézard and G. Parisi, Mean-Field Equations for the Matching and the Travelling Salesman Problems. Euro. Phys. Lett. 2, 913 (1986).

[12] M. Mézard and G. Parisi, The Euclidean Matching Problem. J. Physique 49, 2019 (1988).

[13] R. Brunetti, W. Krauth, M. Mézard and G. Parisi, Extensive Numerical Simulations of Weighted Matchings: Total Length and Distribution of Links in the Optimal Solution. Euro. Phys. Lett. 14, 295 (1991).

[14] G. Parisi, M. and Ratiéville, On the finite size corrections to some random matching problems. Euro. Phys. J. B29, 457 (2002).

[15] W. Krauth and M. Mézard, The cavity method and the travelling salesman problem. Europhys. Lett. 8,213 (1989).

[16] G. Parisi, Constrain optimization and statistical physics. Lectures given at the Varenna summer school. arXiv: cs.CC/0312011

[17] A. Percus, The Traveling Salesman and Related Stochastic Problems, PhD thesis 2007 cond-mat/9803104. Apendix E. Note that in this article $\rho(0)=2$ and consequently they obtain a value for $D_{0}$ which is half of that of the reference [15]

[18] N. J. Cerf, J. Boutet de Monvel, O. Bohigas, O. C. Martin and A. Percus, The Random Link Approximation for the Euclidean Traveling Salesman Problem. J. Phys. France, 7,117 (1997).

[19] R. M. Karp, A pattching algorithm for the non-symmetric traveling salesman problem SIAM Journal on computing 8, 561 (1979).

[20] A. M. Frieze and G. Sorkin, The probabilistic relationship between the assignment and asymmetric traveling salesman problems, Proceedings of the 15th Anual ACM-SIAM Symposium on Discrete Algorithms, Baltimore MD (2001),652-660

[21] A. M. Frieze, On Random symmetric traveling salesman problems, FOCS2002, 789, (2002). and Mathematics of Operations Research, 29, 878 (2004).

[22] J. G. Esteve and F. Falceto, Phase transition in the assignment problem for random matrices. EuroPhys. Lett. 72,691 (2005)

[23] L. Comtet, Advanced Combinatorics: The Art of Finite and Infinite Expansions, rev. enl. ed. Dordrecht, Netherlands: Reidel, 1974, pag 256.

[24] P. Flajolet and R. Sedgewick, Analytic combinatorics, A preliminary version is available at: http://algo.inria.fr/flajolet/Publications/books.html

[25] J. Riordan, An Introduction to Combinatorial Analysis. New York: Wiley, 1980, pag 75. 